\title{
LE SESSE DEL LAGO DI VICO
}

\section{G. Pannocchia}

Nella ormai ampia serie di studi di sesse $\left({ }^{1}\right)$ dei laghi italiani iniziata e guidata da P. Caloi per l'Istituto Nazionale di Geofisica, si può inserire anche quello relativo al lago di Vico.

Anche per questo lago, come già per gli altri, si fa riserva di determinare sperimentalmente $i$ valori delle sesse e di confrontarli con i valori che conseguono dai calcoli teorici, e anche per questo,

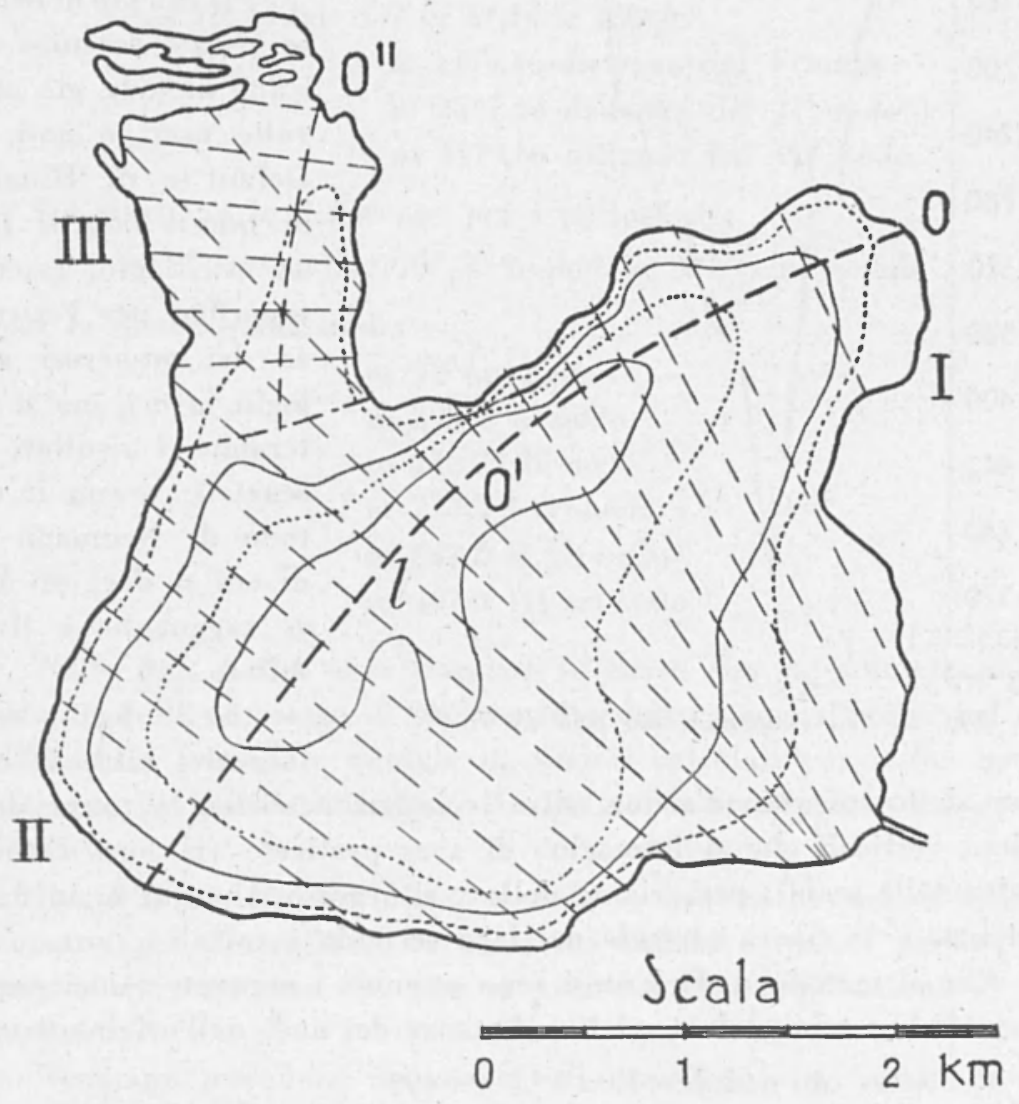

Fig. 1 
come per gli altri, non è da escludere, data la forma piuttosto complessa (fig. 1), che fra i valori calcolati e $i$ valori misurati ci sia una certa divergenza.

Il lago di Vico si trova a circa $12^{\circ} 10^{\prime}$ E. G. e $42^{\circ} 20^{\prime} \mathrm{N}$ ad una altezza s. $1 . \mathrm{m}$. di $507 \mathrm{~m}$; 位o rimane poco a $\mathrm{N}-\mathrm{W}$ del noto centro di Ronciglione. Il suo specchio d'acqua ha una superficie $\mathrm{di} \mathrm{km}^{2} 11.9$ (Atlante dei laghi Italiani G. De Agostini tav. XV); ha una profon-

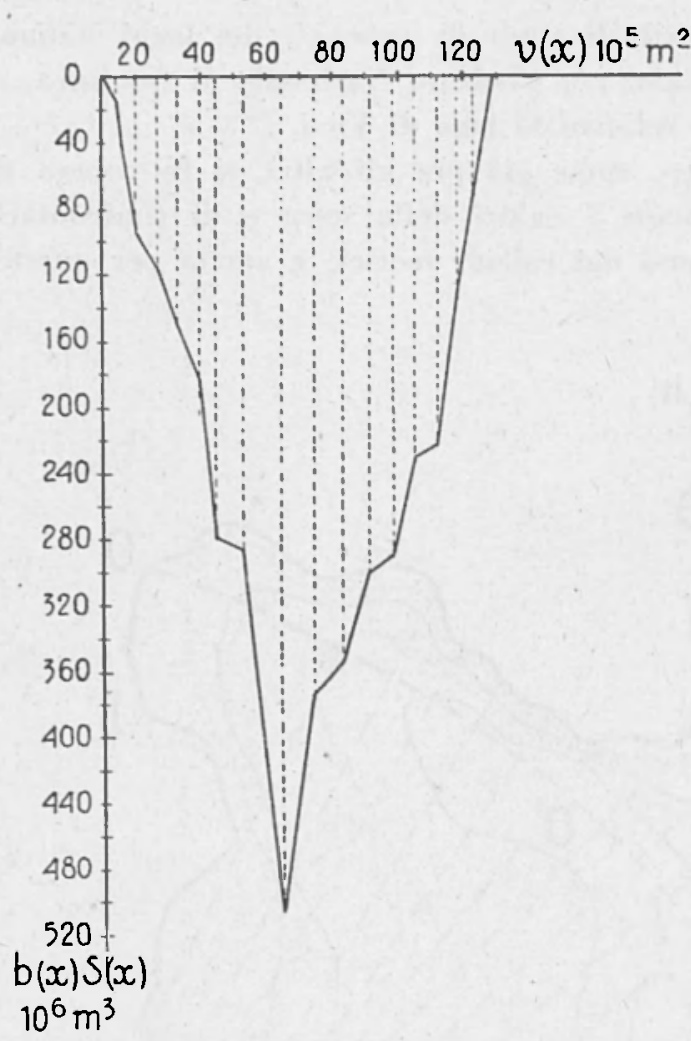

Fig. 2 - Curva normale del lago di Vico dità massima di $4,9,5$ m e media di 19,90 $\mathrm{m}$; una lunghezza secondo la linea 1 (linea di valle) di $4.950 \mathrm{~m}$; il suo volume d'acqua è di circa $268.000 \mathrm{ni}^{3}$.

Il calcolo delle sesse è stato eseguito con i due metodi, già altre volte usati e noti, di Defant e di Hidaka; di questi non si farà un richiamo essendo superfluo per l'esistenza dei numerosi analoghi lavori, ma si noteranno i risultati essenziali; e con il metodo di Neumann ( $\left.{ }^{3}\right)$ di cui si darà un breve ragguaglio e il risultato.

Nella fig. 1 sono indicate, oltre il contorno dello specchio d'acqua, oltre le isobatimetriche, le tracce delle sezioni verticali che si immagina di aver praticato (la loro distanza risulta dalla scala); per origine delle $x$ si è preso il punto 0 ; in fig. 2 à riportata la curva normale del lago secondo i calcoli.

Con il metodo di Defant si sono ottenuti i seguenti valori per le sesse uni-bi-e tri-nodale e relative distanze dei nodi dall'origine 0 :

sessa uni-nodale: $T=8^{\mathrm{m}}, 3=498^{\mathrm{s}}$

m 2250 distanza del I nodo; 
sessa bi-nodale : $T=5^{\mathrm{m}}, 11=306^{\mathrm{s}}, 6$

m 1000 distanza del I nodo

m 3250 distanza del II nodo;

sessa tri-nodale : $T=3^{\mathrm{m}}, 67=220^{\mathrm{s}}, 2$

n) 460 distanza del I nodo

m 2000 distanza del II nodo

m 3712,5 distanza del III nodo.

Con il metodo di Hidaka si sono ottenuti i seguenti valori:

sessa uni-nodale: $T=7^{\mathrm{m}}, 68=460^{\mathrm{s}}, 6$

m 2342,44 distanza del I nodo;

sessa bi-nodale : $T=4^{\mathrm{m}}, 71=282^{\mathrm{s}}, 9$

m 1393,35 distanza del I nodo

nl 3480,20 distanza del II nodo;

sessa tri-nodale : $T=3^{\mathrm{m}}, 14=188^{\mathrm{s}}, 6$

m 1173,46 distanza del I nodo

n 2547,58 distanza del II nodo

m 4119,56 distanza del III nodo.

Ne risultano le differenze per $i$ periodi di:

$0^{\mathrm{m}}, 62$ uni-nodale; $0^{\mathrm{m}}, 40$ bi-nodale; $0^{\mathrm{m}}, 53$ tri-nodale

e per le distanze dei nodi:

$$
\begin{aligned}
& \text { m } 92 \text { uni-nodo } \\
& \text { m } 393 \text { I bi-nodo } \\
& \text { m } 230 \text { II bi-nodo } \\
& \text { m } 713,5 \text { I tri-nodo } \\
& \text { m } 547,6 \text { II tri-nodo } \\
& \text { m } 407,0 \text { III tri-nodo. }
\end{aligned}
$$

Nelle figg. 3 e 4 sono tracciate le curve che rappresentano l'andamento delle ampiezze di spostamento della massa d'acqua nel caso dell'uni-bi e tri-nodale desunte dai valori calcolati per i due metodi citati (curva continua metodo di Defant e tratteggiata metodo di Hidaka).

Come si è detto all'inizio, si è fatto uso anclie del metodo di Neumann per determinare la sessa uni-nodale e questo perché si è ritenuto che la forma del lago si prestasse alla applicazione di tale metodo.

Neumann introduce il concetto di impedenza, già usato in acustica e nei circuiti oscillanti elettromagnetici, per i sistemi continui, 


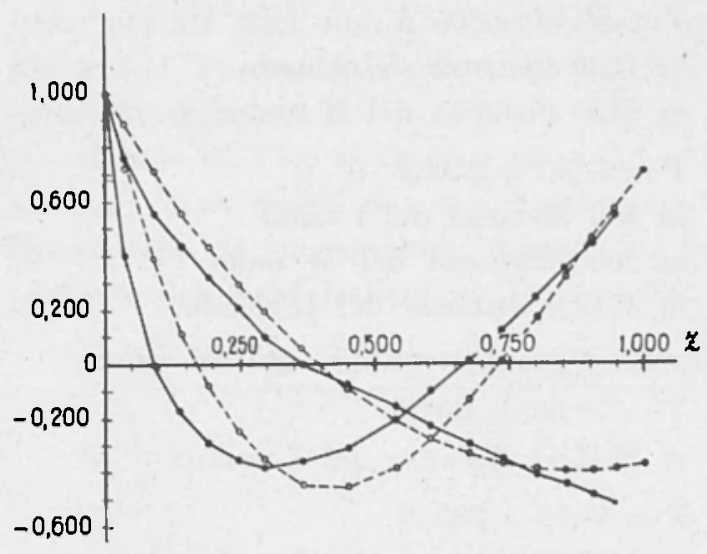

Fig. 3

applicandolo quindi alla teoria delle sesse. Il Neumann tien conto dell'influenza reciproca di bacini comunicanti per mezzo di canali sul periodo complessivo d'oscillazione; o di quella di vari rami di uno stesso hacino. Per impendenza idrodinamica nelle oscillazioni di masse di acqua egli definisce il rapporto:

$$
\vec{Z}=\frac{p_{0}}{S\left(\partial \zeta_{0} / \partial t\right)_{\max }}=\frac{\text { ampiezza di pressione }}{\text { superficie } X \text { ampiezza di velocità }}
$$

e ne calcola il valore attraverso le equazioni idrodinamiche per i casi più comuni. Si riporta qui solo il valore che essa assume, secondo il

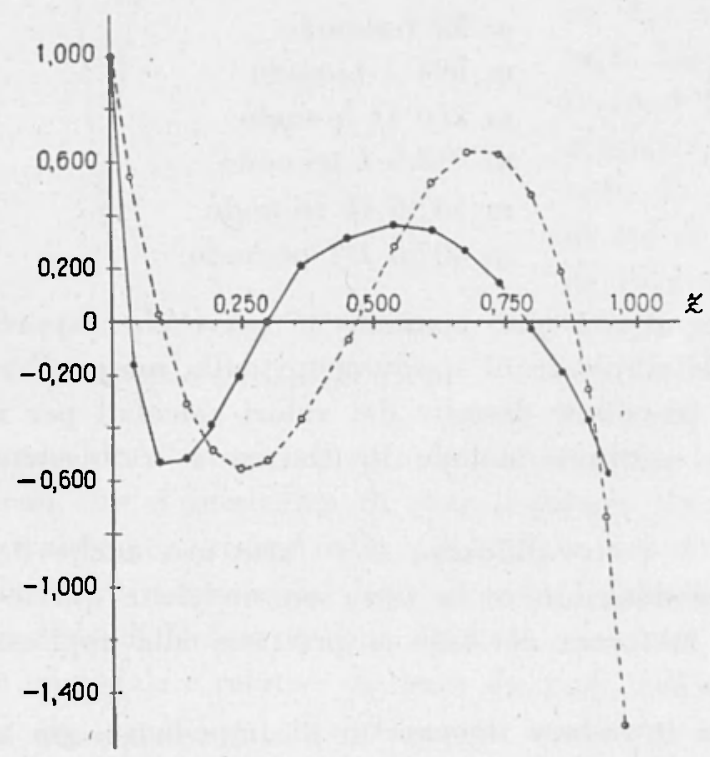

Fig. 4 
Neumann, in caso di bacini chiusi da un solo lato dalla superficie trasversale $S=b h$ ( $b=$ larghezza, $h=$ profondità):

$$
Z=-\frac{i_{\varrho} c}{S} \operatorname{cotg} \frac{(1) l}{c}, L=\lambda / 4
$$

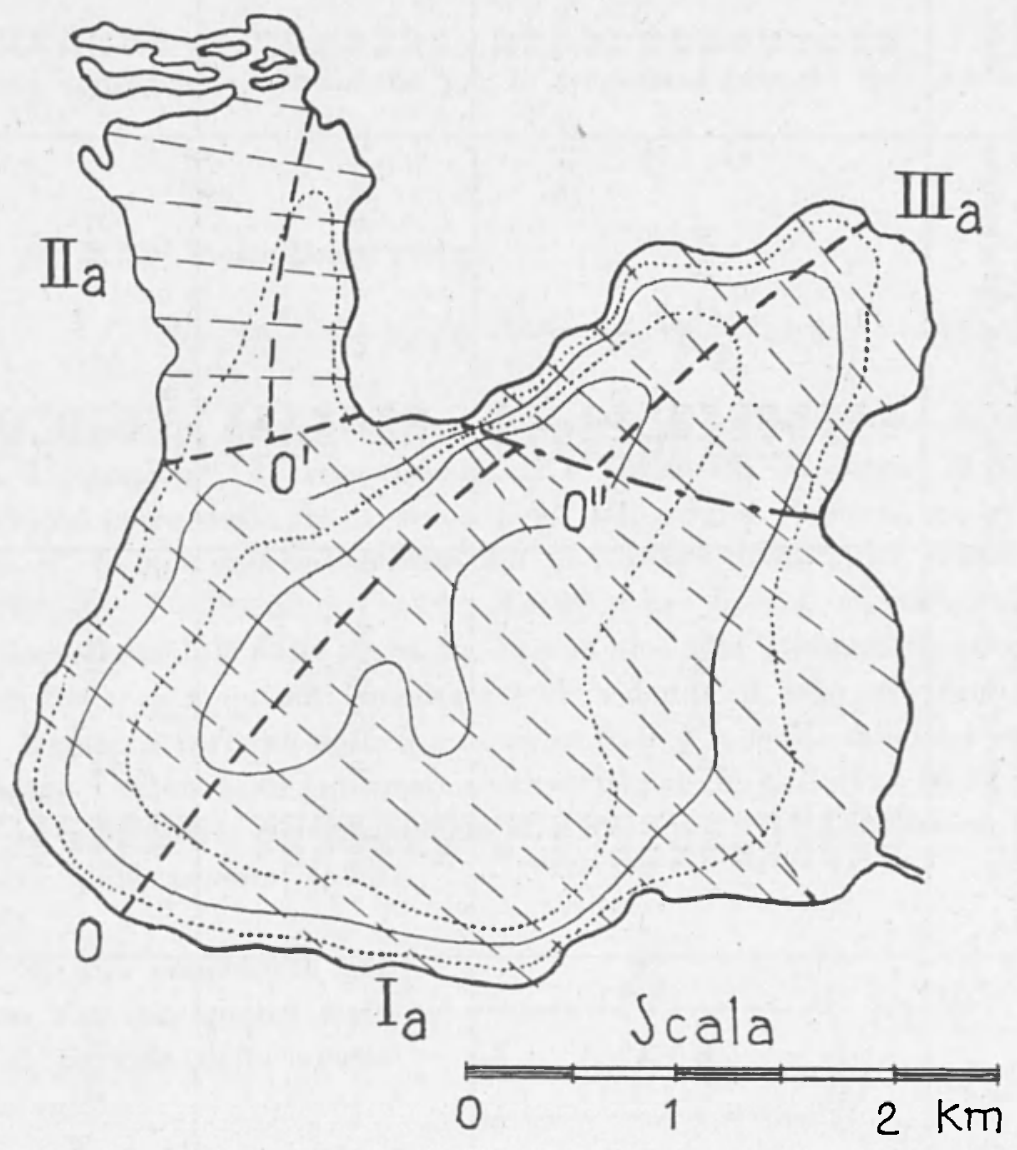

Fig. 5

dove $i=\overline{-1}, c=l \overline{g h}, \omega=\frac{2 \pi}{T}, L=$ lunghezza del bacino, $\lambda=$ lunghezza d'onda, $\varrho=$ densità dell'acqua.

Sempre per Neumann per un lago $I$, simile al caso del lago di Vico, che si ramifichi in $I I$ e $I I I$, i due rami $I I$ e $I I I$ vengono a trovarsi in parallelo dopo il ramo $I$ di impedenza $Z$. Sicché analogamente ai circuiti elettromagnetici l'impedenza complessiva di $I I$ e $I I I$ sarà: 
Tabella I

\begin{tabular}{|c|c|c|c|c|c|}
\hline $\begin{array}{c}\text { "amo } \\
\text { del } \\
\text { lago }\end{array}$ & 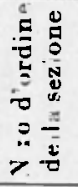 & $\begin{array}{l}\text { Terea delle sezioni verticali } \\
\text { in } \mathrm{m} .2\end{array}$ & $\begin{array}{l}\text { barghezza alla superlicie } \\
\text { della sezione in n. }\end{array}$ & $\begin{array}{cl}\text { Distanza progressiva } \\
\text { dall'origine in } \\
\text { m. della sezione }\end{array}$ & $\begin{array}{l}\mathrm{h} \\
\text { Profondità max } \\
\text { della sez. in in. }\end{array}$ \\
\hline$I_{a}$ & $\begin{array}{l}9 \\
8.5 \\
8 \\
7.5 \\
7 \\
6.5 \\
6 \\
5.5 \\
5 \\
4.5 \\
4 \\
3 \\
2 \\
1\end{array}$ & $\begin{array}{l}38.800 \\
58.250 \\
83.000 \\
83.300 \\
97.950 \\
97.5 \div 0 \\
91.0 \div 0 \\
82.150 \\
92.000 \\
72.00 \\
66.850 \\
54.400 \\
36.500 \\
10.400\end{array}$ & $\begin{array}{l}175 \\
2225 \\
2685 \\
2775 \\
2950 \\
305 \\
3150 \\
3175 \\
3175 \\
2825 \\
275 \\
2100 \\
1750 \\
950\end{array}$ & $\begin{array}{r}450 \\
700 \\
950 \\
1200 \\
1450 \\
1700 \\
19: 0 \quad T^{1} \\
2200 \\
240 \\
2700 \\
2775 \\
282 i \\
3075 \\
3300\end{array}$ & $\begin{array}{l}30 \\
34 \\
41 \\
42 \\
42 \\
42 \\
45 \\
43 \\
43 \\
41 \\
43 \\
45 \\
43 \\
43 \\
25\end{array}$ \\
\hline 11 & $\begin{array}{l}7 \\
6 \\
5 \\
4 \\
3 \\
2 \\
1\end{array}$ & $\begin{array}{r}11.200 \\
8.250 \\
7.750 \\
7.450 \\
6.800 \\
3.850 \\
7: 0\end{array}$ & $\begin{array}{r}1000 \\
875 \\
925 \\
1050 \\
1075 \\
1275 \\
375\end{array} \quad{ }_{11 \mathrm{a}}=939.29$ & $\begin{array}{r}2: 0 \\
5 \% 0 \\
50 \\
1000 \\
1250 \\
1,00 \\
1950\end{array} T_{11 \mathrm{a}}=19=0$ & 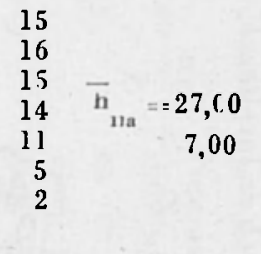 \\
\hline $11 \mathrm{l}_{\mathrm{a}}$ & $\begin{array}{l}1 \\
2 \\
3 \\
4 \\
5 \\
6\end{array}$ & $\begin{array}{r}30.60 \\
39.050 \\
35.200 \\
22.10 ! \\
11.500 \\
7.800\end{array} \bar{\sigma}=24.883$ & $\begin{array}{r}850 \\
1500 \\
1475 \\
1175 \\
1000 \\
975\end{array} \overline{\mathrm{b}}_{\text {111а }}=1162.5$ & $\begin{array}{r}15 \\
425 \\
675 \\
925 \\
115 \\
1425 \\
1675\end{array} \quad T_{11 \mathrm{a}}=16: 5$ & $\begin{array}{l}40 \\
40 \\
35 \\
25 \\
20 \\
13\end{array} \bar{h}_{\text {H1. }}=21.40$ \\
\hline
\end{tabular}




$$
\frac{1}{P}=\frac{1}{\eta_{0}}+\frac{1}{Z_{3}} \quad \text { da cui } \quad P=\frac{Z_{2} Z_{3}}{Z_{2}+Z_{3}}
$$

Ed essendo il ramo $I$ in serie con $(I I+I I I)$ (fig. 6):

$$
Z_{1}+P=Z_{1}+\frac{Z_{2} \quad Z_{3}}{Z_{2}+Z_{3}}
$$

da cui consegue la condizione per la frequenza propria dell'insieme:

$$
\frac{1}{Z_{1}}+\frac{1}{Z_{2}}+\frac{1}{Z_{3}}=0
$$

che per la [2] moltiplicata per $g$ :

$$
b_{1} c_{1} \operatorname{tg} \frac{\omega I_{1}}{c_{1}}+b_{2} c_{2} \operatorname{tg} \frac{\omega L_{2}}{c_{2}}+b_{3} c_{3} \operatorname{tg} \frac{\omega L_{3}}{c_{3}}=0
$$

L'equazione, nota Neumann, coincide con quella dedotta da Zei. lon. L'equazione [3] trascendente si è cimentata dapprima al lago suddiviso come dalla fig. 1 (rami I. II. III, origine delle $x_{1}, x_{0}, x_{3}$ in $0,0^{\prime}, 0^{\prime \prime}$ usando successivamente per $\omega$ i valori dedotti dai valori di $T=8^{\mathrm{m}}, 3 ; T-7^{\mathrm{m}}, 68 ; T=7^{\mathrm{m}} ; T=9^{\mathrm{m}}$ e per $b_{\mathrm{i}}, c_{\mathrm{i}}, l_{\mathrm{i}}\left(i=1^{0}, 2^{0}, 3^{0}\right)$ i valori deducibili dalla carta fig. 1 ; non si è mai ottenuto di poterla soddisfare; si è quindi cimentata suddividendo il lago nei rami $l_{a}$, $I I_{\mathrm{a}}, I I I_{\mathrm{a}}$ fig. 5 (origine delle $x_{1}, x_{2}, x_{3}$ in $0,0^{\prime} 0^{\prime \prime}$ ). Nella tabella $I$ sono riportati i valori usati (misurati o calcolati) per $b_{i}, c_{i}, l_{i}\left(i=1^{0}, 2^{0}, 3^{0}\right)$. Per $\omega=0,011081$, conseguente da $T=9^{\mathrm{m}}, 45=567^{\mathrm{s}}$, l'equazione [3] risulta praticamente soddisfatta.

Si può concludere per. tanto che con questo metodo il periodo della uni-nodale vale:

$$
T=9^{\mathrm{m}}, 45 .
$$

Dei tre periodi per la

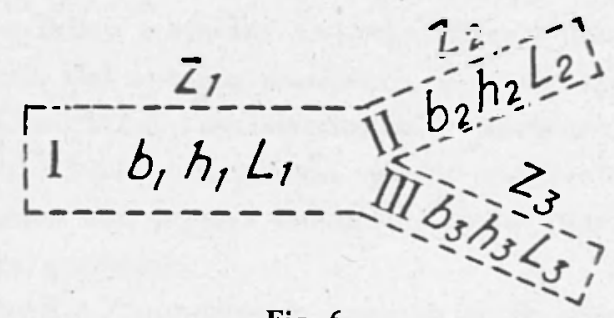

Fig. 6 uni-nodale trovati per via teorica non si può dire a priori quale sia il più prossimo alla realtà; per altro si ritiene che quest'ultimo sia il più attendibile sembrando molto suasive le induzioni e anche le misure e calcoli fatti dal Neumann; per altro la misura diretta dirà l'ultima parola.

$$
\text { Roma - Istituto Nazionale di Geofisica - Giugno } 1950 .
$$




\section{RIASSUNTO}

Nel presente lavoro si determinano con $i$ metodi di Defant e di Hidaka $i$ periodi delle sesse uni-bi e tri-nodale del lago di Vico, le relative distanze dei nodi e le curve rappresentanti l'andamento delle ampiezze di oscillazione con $i$ metodi detti. Con il metodo indicato da Neumann si determina di nuovo il periodo della sessa uni-nodale.

\section{BIBLIOGR AFIA}

(1) Calor P.: Le sesse del lago di Garda. Parte primil. Sesse che interessano l'intero lago. Ann. Geof., I, 24-48 (1948).

Calor P.: Le sesse del lago di Garda. Parte secondiı. Ann. Geof., I, 175-199 (1948).

(2) Perrone: Carta idrografica d'Italia. Vol. Tevere, 256-258.

(3) Neumann G.: Beitrage zur Theorie der Seiches in zusammengesetzten Seebeckensystemen. Ann. Geof., III, 15.24 opp. $11-116$ (1950). 